\title{
A NOTE ON MARKOV BRANCHING PROCESSES
}

\author{
FRED M. HOPPE, ${ }^{*}$ The University of Michigan, Ann Arbor
}

\begin{abstract}
We present a simple proof of Zolotarev's representation for the Laplace transform of the normalized limit of a Markov branching process and relate it to the Harris representation.
\end{abstract}

GALTON-WATSON: BACKWARD KOLMOGOROV EQUATION; LAPLACE TRANSFORM

Let $Z(t)$ denote a supercritical Markov branching process with infinitesimal generating function $u(x)=a(f(x)-x)$ where $f(x)=\sum f_{i} x^{i}$ is the offspring p.g.f. with mean $m$ and where $a^{-1}$ is the mean particle lifetime.

Zolotarev (1957) showed the existence of a normalizing function $\gamma(t)$ making $\gamma(t) Z(t)$ converge in distribution to a proper limit $W$ whose Laplace transform $\phi(s)=$ $E[\exp (-s W) \mid Z(0)=1]$ satisfies

$$
\phi^{-1}(x)=\exp \int_{\Delta}^{x} \frac{m-1}{f(r)-r} d r
$$

for a specified constant $\Delta$.

Here is a very quick proof of (1) relying on the Poincaré functional equation (familiar in the Galton-Watson setting) for the embedded discrete skeleton. Integrate the backward Kolmogorov equation $\partial F / \partial t=u(F(x, t))$ to obtain

$$
t=\int_{\Delta}^{F(\Delta, t)} \frac{d r}{u(r)}
$$

where $F(x, t)=E\left[x^{Z(t)} \mid Z(0)=1\right]$ and $\Delta$ is an arbitrary constant. For each $t$ the process $\{Z(n t), n \geqq 0\}$ is Galton-Watson so, using the constants of Seneta (1968), we find that

$$
\phi(\exp (\lambda t) s)=F(\phi(s), t)
$$

where $\lambda=a(m-1)$ is the Malthusian parameter. Setting $s=1$ and defining $\Delta=\phi(1)$ we get $F(\Delta, t)=\phi(\exp \lambda t)$ which, when substituted into (2) with $x=\phi(\exp \lambda t)$, results in

$$
\phi^{-1}(x)=\exp \lambda t=\exp \lambda \int_{\Delta}^{x} \frac{d r}{u(r)}=\exp \int_{\Delta}^{x} \frac{m-1}{f(r)-r} d r,
$$

completing the proof.

Next, we separate the integral in (1) into a sum

$$
\int_{\Delta}^{x}\left(\frac{m-1}{f(r)-r}+\frac{1}{1-r}\right) d r-\int_{\Delta}^{x} \frac{1}{1-r} d r
$$

\footnotetext{
Received 2 January 1985.

* Postal address: Department of Statistics, The University of Michigan, Ann Arbor, MI 48109,
} USA. 
If (and only if) the familiar logarithmic moment condition $\sum(i \log i) f_{i}<\infty$ holds, the first integral is known to remain bounded as $x \rightarrow 1$ (essentially Theorem 4.3 of Seneta (1969)) whence it is permissible to split the integration into one part running from $\Delta$ to 1 and a second part from 1 to $x$, which results in

$$
\int_{1}^{x}\left(\frac{m-1}{f(r)-r}+\frac{1}{1-r}\right) d r-\int_{1}^{\Delta}\left(\frac{m-1}{f(r)-r}+\frac{1}{1-r}\right) d r+\log \frac{1-x}{1-\Delta}
$$

and culminates in

$$
\phi^{-1}(x)=(1-x) \exp \int_{1}^{x}\left(\frac{m-1}{f(r)-r}+\frac{1}{1-r}\right) d r /(1-\Delta) \exp \int_{1}^{\Delta}\left(\frac{m-1}{f(r)-r}+\frac{1}{1-r}\right) d r .
$$

Under the classical norming $W=\lim \exp (-\lambda t) Z(t)$ and $E[W]=1$, and hence $\lim (x \rightarrow 1) \phi^{-1}(x) /(1-x)=1$. This shows that the denominator in the previous line equals 1 and therefore

$$
\phi^{-1}(x)=(1-x) \exp \int_{1}^{x}\left(\frac{m-1}{f(r)-r}+\frac{1}{1-r}\right) d r,
$$

a representation first derived by Harris (1951) assuming the finiteness of the second moment $\sum i^{2} f_{i}$. There is a proof by Karlin and McGregor (1968) also only requiring $\sum(i \log i) f_{i}<\infty$ which is based on a different approach.

I would like to thank E. Seneta for the Zolotarev reference.

\section{References}

HARRIS, T. E. (1951) Some mathematical models for branching processes. Proc. 2nd Berkeley Symposium on Mathematical Statistics and Probability, pp 305-328.

KARLIN, S. AND MCGREGOR, J. (1968) Embeddability of discrete time simple branching processes into continuous time branching processes. Trans. Amer. Math. Soc. 132, 115-136.

SENETA, E. (1968) On recent theorems concerning the supercritical Galton-Watson process. Ann. Math. Statist. 39, 2098-2102.

Seneta, E. (1969) Functional equations and the Galton-Watson process. Adv. Appl. Prob. 1, $1-42$.

Zolotarev, V. M. (1957) More exact statements of several theorems in the theory of branching processes. Theory Prob. Appl. 2, 245-253. 